\title{
UPCommons
}

Portal del coneixement obert de la UPC

http://upcommons.upc.edu/e-prints

This is a post-peer-review, pre-copy edit version of an article published in Meccanica. The final authenticated version is available online at: https://doi.org/10.1007/s11012-018-0889-0.

\section{Published paper:}

Quintanilla, R. Some qualitative results for a modification of the Green-Lindsay thermoelasticity. "Meccanica", Novembre 2018, vol. 53, núm. 14, p. 3607-3613. doi:10.1007/s11012-018-0889-0

URL d'aquest document a UPCommons E-prints:

https://upcommons.upc.edu/handle/2117/122738 


\title{
Some qualitative results for a modification of the Green-Lindsay thermoelasticity
}

\author{
R. Quintanilla \\ Dep. Matemàtiques, ESEIAAT, Universitat Politècnica de Catalunya \\ Colom, 11. Terrassa (08222). Barcelona. Spain \\ E-mail address: Ramon.Quintanilla@upc.edu
}

\begin{abstract}
In this short note we consider a recent modification of the Green-Lindsay thermoelastic theory proposed at [10]. We consider a functional defined on the solutions of the problem. It allows us to obtain the continuous dependence of the solutions with respect to the initial conditions and to the supply terms, the time exponential decay of solutions and an alternative of Phragmén-Lindelöf type for the spatial behaviour.

keywords: Modified Green-Lindsay thermoelasticity, Continuous dependence, Uniqueness, Exponential decay, Spatial behaviour
\end{abstract}

\section{Introduction}

It is known that the classical formulation of the Fourier law combined with the classical energy equation ${ }^{1}$

$$
c_{E} \dot{\theta}=-q_{i, i},
$$

brings to the paradox of the infinite speed of propagation. For this reason many people has been interested to overcome this difficulty and to propose alternative theories which were free of this paradox. In this sense we can cite the hyperbolic proposition of Cattaneo for the heat conduction [2] or the alternative propositions of Green and Naghdi $[6,7]$. We can recall two extensions of the Cattaneo law to the thermoelasticity. One corresponds to the theory of Lord and Shulman [14] and the second is the theory of Green and Lindsay [5]. This last one is based in a generalized dissipation inequality by considering a scalar function depending upon the temperature and its rate. In this short note we are going to be involved with a recent modification of this theory proposed in [10]. In that paper the authors introduce a second order tensor depending on the strain and its rate and a generalized Clausius-Duhem inequality. They propose an alternative system of equations (see (2.1), (2.2)).

In this note we are interested in the study of the qualitative behavior of the solutions of this new system of equations. To avoid technical difficulties we are going to

\footnotetext{
${ }^{1}$ Here $\theta$ is the relative temperature and $q_{i}$ is the heat flux vector
} 
assume the existence of solutions and we center our attention to several qualitative properties. We want to propose a functional defined on the solutions which is very useful in the study of the system. We believe that this is the main contribution of this paper. With the help of this functional we can use the energy methods and we can seen the continuous dependence of the solutions with respect to the initial data and to the supply terms. Later we also prove the exponential decay of solutions and we continue by giving a description of the spatial behaviour of the solutions ${ }^{2}$. It is worth recalling that continuous dependence, uniqueness, exponential decay and spatial stability are basic properties deserving to be studied for every thermoelastic theory. In this sense, it is natural to find results of this kind for several thermoelastic theories. We can cite several papers devoted to this kind of studies $[1,3,8,9,11,12,15,16,17,18]$.

In the next section we recall the system of equations with we are going to work and the initial and boundary conditions for the problem. Later in Section 3 we prove a result on the continuous dependence of the solutions with respect to the initial conditions and to the supply terms. In Section 4 we show the exponential stability of the solutions with respect to the time in the case where the supply terms vanish. We finish in Section 5 by proving a Phragmén-Lindelöf alternative for the spatial behaviour of the solutions.

\section{Equations and assumptions}

We now propose the initial-boundary-value problem to be studied in the next two sections. We consider a bounded domain $B$ in the three-dimensional Euclidean space such that the boundary is smooth enough to apply the divergence theorem.

We recall that the system of field equations was obtained in [10]. We have

$$
\begin{gathered}
\rho \ddot{u}_{i}=\left(C_{i j k l}\left(u_{k, l}+\tau_{1} \dot{u}_{k, l}\right)-\gamma_{i j}\left(\theta+\tau_{1} \dot{\theta}\right)\right)_{, j}+b_{i} \\
\gamma_{i j} \theta_{0}\left(\dot{u}_{i, j}+\tau_{0} \ddot{u}_{i, j}\right)+\rho c_{E}\left(\dot{\theta}+\tau_{0} \ddot{\theta}\right)=\left(k_{i j} \theta_{, i}\right)_{, j}+r
\end{gathered}
$$

Here $\rho$ is the mass density, $C_{i j k l}$ is the elasticity tensor that satisfies the symmetry

$$
C_{i j k l}=C_{k l i j}
$$

$\gamma_{i j}$ is the thermal expansion tensor, $c_{E}$ is the thermal capacity, $k_{i j}$ is the thermal conductivity tensor which it is also symmetric

$$
k_{i j}=k_{j i}
$$

$\theta_{0}$ is the uniform absolute temperature in the reference configuration, $b_{i}$ and $r$ are the supply terms, $\left(u_{i}\right)$ is the displacement vector, $\theta$ is the relative temperature and $\tau_{0}, \tau_{1}$ are two parameters that satisfy (see [10], eq. 22)

$$
\tau_{1}>\tau_{0}>0 \text {. }
$$

In the system of equations and from now on the notation ", $i$ " means derivation with respect to the direction $x_{i}$, a superposed dot means the time derivative and the repetition in the indices means summation on the corresponding index.

\footnotetext{
${ }^{2}$ It is worth recalling that the spatial stability of solutions for partial differential equations is related with the well-known Saint-Venant's principle in thermomechanics [19, 20]
} 
For what follows it is useful to have the system of equations in terms of dimensionless quantities. We introduce the variables:

$$
u_{i}^{\prime}=\frac{u_{i}}{L}, \quad x_{i}^{\prime}=\frac{x_{i}}{L}, t^{\prime}=\frac{t}{t_{0}}, \quad \theta^{\prime}=\frac{\theta}{K}, \quad \theta_{0}^{\prime}=\frac{\theta_{0}}{K}, \quad \rho^{\prime}=\frac{\rho L^{3}}{m_{0}}, \quad \tau_{0,1}^{\prime}=\frac{\tau_{0,1}}{t_{0}},
$$

where $L, t_{0}, m_{0}$ and $K$ be four constants with dimensions of length, time, mass and temperature respectively. Furthermore, we introduce the following notations

$$
C_{i j k l}^{\prime}=\frac{t_{0}^{2} L}{m_{0}} C_{i j k l}, \quad \gamma_{i j}^{\prime}=\frac{t_{0}^{2} K L}{m_{0}} \gamma_{i j}, \quad c_{E}^{\prime}=\frac{t_{0}^{2} K}{L^{2}} c_{E}, \quad k_{i j}^{\prime}=\frac{K t_{0}^{3}}{L m_{0}} k_{i j} .
$$

A similar thing can be done for $b_{i}$ and $r$. We can write our system in the new variables. We will obtain the same equations, but in this case the variables are dimensionless. For simplicity we omit the colon. Therefore, we will study our system in the convention that we work with dimensionless variables.

To simplify the calculations, but without loss of generality we assume that the uniform absolute temperature in the reference configuration is equal to 1 .

To determine the initial-boundary-value problem we will study in Sections 3 and 4 we need to impose the initial and boundary conditions. We assume the initial conditions

$$
u_{i}(\mathbf{x}, 0)=u_{i}^{0}(\mathbf{x}), \dot{u}_{i}(\mathbf{x}, 0)=v_{i}^{0}(\mathbf{x}), \theta(\mathbf{x}, 0)=\theta^{0}(\mathbf{x}), \dot{\theta}(\mathbf{x}, 0)=T^{0}(\mathbf{x}), \mathbf{x} \in B .
$$

We consider null Dirichlet boundary conditions

$$
u_{i}(\mathbf{x}, t)=\theta(\mathbf{x}, t)=0, \mathbf{x} \in \partial B, t>0 .
$$

Apart that we assume that all the constitutive tensors are bounded in this note we are going to suppose the following conditions:

(i) Mass density and heat capacity are strictly positive. That is

$$
\rho(\mathbf{x}) \geq \rho_{1}>0, \quad c_{E}(\mathbf{x}) \geq c>0 .
$$

(ii) There exists a positive constant $C_{1}$ such that

$$
C_{i j k l} \xi_{i j} \xi_{k l} \geq C_{1} \xi_{i j} \xi_{i j}
$$

for every tensor $\left(\xi_{i j}\right)$.

(iii) There exists a positive constant $k_{1}$ such that

$$
k_{i j} \xi_{i} \xi_{j} \geq k_{1} \xi_{i} \xi_{i}
$$

for every vector $\xi_{i}$.

The meaning of the assumptions in (i) is clear. Condition (ii) can be understand in terms of the elastic stability and condition (iii) is related with the well-known property of a heat conductor (see also [10], eq. 19). 


\section{Continuous dependence}

The aim of this section is to give a continuous dependence result for the solutions of the problem determined by $(2.1),(2.2),(2.6),(2.7)$. It is worth remarking that uniqueness of solutions will be a consequence of the continuous dependence in this case.

We first multiply (2.1) by $\dot{u}_{i}+\tau_{0} \ddot{u}_{i}$, integrate over $B$, apply the boundary conditions and after the use of the divergence theorem we obtain that

$$
\begin{gathered}
\int_{B} \rho \ddot{u}_{i}\left(\dot{u}_{i}+\tau_{0} \ddot{u}_{i}\right) d v=-\int_{B} C_{i j k l}\left(\dot{u}_{i, j}+\tau_{0} \ddot{u}_{i, j}\right)\left(u_{k, l}+\tau_{1} \dot{u}_{k, l}\right) d v \\
+\int_{B} \gamma_{i j}\left(\theta+\tau_{1} \dot{\theta}\right)\left(\dot{u}_{i, j}+\tau_{0} \ddot{u}_{i, j}\right) d v+\int_{B} b_{i}\left(\dot{u}_{i}+\tau_{0} \ddot{u}_{i}\right) d v .
\end{gathered}
$$

Now we multiply (2.2) by $\theta+\tau_{1} \dot{\theta}$ integrate over $B$, apply the boundary conditions and after the use of the divergence theorem we get that

$$
\begin{gathered}
\int_{B} \gamma_{i j}\left(\theta+\tau_{1} \dot{\theta}\right)\left(\dot{u}_{i, j}+\tau_{0} \ddot{u}_{i, j}\right) d v+\int_{B} \rho c_{E}\left(\theta+\tau_{1} \dot{\theta}\right)\left(\dot{\theta}+\tau_{0} \ddot{\theta}\right) d v \\
=-\int_{B} k_{i j} \theta_{, i}\left(\theta_{, j}+\tau_{1} \dot{\theta}_{, j}\right) d v+\int_{B} r\left(\theta+\tau_{1} \dot{\theta}\right) d v
\end{gathered}
$$

In the next step of the study it is suitable to take into account the following equalities:

$$
\int_{B} C_{i j k l} \ddot{u}_{i, j} u_{k, l} d v=\frac{d}{d t} \int_{B} C_{i j k l} \dot{u}_{i, j} u_{k, l} d v-\int_{B} C_{i j k l} \dot{u}_{i, j} \dot{u}_{k, l} d v,
$$

and

$$
\int_{B} \ddot{\theta} \theta d v=\frac{d}{d t} \int_{B} \dot{\theta} \theta d v-\int_{B}|\dot{\theta}|^{2} d v .
$$

Therefore from (3.1)-(3.4) we can see that if we consider the function

$$
\begin{aligned}
& E(t)=\frac{1}{2} \int_{B}\left(\rho \dot{u}_{i} \dot{u}_{i}+C_{i j k l} u_{i, j} u_{k, l}+\tau_{0} \tau_{1} C_{i j k l} \dot{u}_{i, j} \dot{u}_{k, l}+\rho c_{E} \theta^{2}\right. \\
& \left.+\tau_{0} \tau_{1} \rho c_{E}|\dot{\theta}|^{2}+\tau_{1} k_{i j} \theta_{, i} \theta_{, j}+2 \tau_{0} C_{i j k l} \dot{u}_{i, j} u_{k, l}+2 \rho c_{E} \tau_{0} \theta \dot{\theta}\right) d v
\end{aligned}
$$

we obtain that

$$
\begin{gathered}
\dot{E}(t)=-\int_{B}\left(\tau_{0} \rho \ddot{u}_{i} \ddot{u}_{i}+\left(\tau_{1}-\tau_{0}\right) C_{i j k l} \dot{u}_{i, j} \dot{u}_{k, l}\right. \\
\left.+\left(\tau_{1}-\tau_{0}\right) \rho c_{E}|\dot{\theta}|^{2}+k_{i j} \theta_{, i} \theta_{, j}-b_{i}\left(\dot{u}_{i}+\tau_{0} \ddot{u}_{i}\right)-r\left(\theta+\tau_{1} \dot{\theta}\right)\right) d v .
\end{gathered}
$$

It is worth noting that the matrix

$$
\left(\begin{array}{cc}
1 & \tau_{0} \\
\tau_{0} & \tau_{0} \tau_{1}
\end{array}\right)
$$

is positive definite because of the condition (2.5). Thus the function $E(t)$ considered at (3.5) defines a measure on the solutions of the problem. 
After the use of the arithmetic-geometric mean inequality it is easy to see the existence of four constants $D_{i}, i=1 \ldots 4$ (using the Poincaré inequality when it is needed) such that

$$
\begin{gathered}
\int_{B} b_{i} \dot{u}_{i} d v \leq\left(\tau_{1}-\tau_{0}\right) \int_{B} C_{i j k l} \dot{u}_{i, j} \dot{u}_{k, l} d v+D_{1} \int_{B} b_{i} b_{i} d v \\
\tau_{0} \int_{B} b_{i} \ddot{u}_{i} d v \leq \tau_{0} \int_{B} \rho \ddot{u}_{i} \ddot{u}_{i} d v+D_{2} \int_{B} b_{i} b_{i} d v \\
\int_{B} r \theta d v \leq \int_{B} k_{i j} \theta_{, i} \theta_{, j} d v+D_{3} \int_{B} r^{2} d v \\
\tau_{1} \int_{B} r \dot{\theta} d v \leq\left(\tau_{1}-\tau_{0}\right) \int_{B} \rho c_{E}|\dot{\theta}|^{2} d v+D_{4} \int_{B} r^{2} d v
\end{gathered}
$$

In view of $(3.6),(3.8)-(3.11)$ we see that

$$
\dot{E}(t) \leq C \int_{B}\left(b_{i} b_{i}+r^{2}\right) d v
$$

where $C=\max \left(D_{1}+D_{2}, D_{3}+D_{4}\right)$.

After an integration in (3.12) we see that

$$
E(t) \leq E(0)+C \int_{0}^{t} \int_{B}\left(b_{i} b_{i}+r^{2}\right) d v d s
$$

Therefore we have proved:

Theorem 3.1. Let $\left(u_{i}, \theta\right)$ be a solution of the initial-boundary-value problem determined by the system (2.1), (2.2), the initial condition (2.6) and the boundary conditions (2.7). Then, the solutions satisfy the estimate (3.13) where the function $E(t)$ is defined at (3.5).

Now we will see the uniqueness of solutions to our problem. It will be sufficient to prove that the only solution for the problem determined by null initial solutions when the supply terms vanish is the null solution. We note that in this case the estimate (3.13) implies that

$$
E(t) \leq 0,
$$

for every $t>0$. In view of the definition of the function $E(t)$ we see that (3.14) implies that $\left(u_{i}, \theta\right)=(0,0)$ for every $t \geq 0$. Therefore we can conclude that:

Theorem 3.2. The initial-boundary-value problem determined by the system (2.1), (2.2), the initial condition (2.6) and the boundary conditions (2.7) has uniqueness of solutions.

We note that assumption (ii) is usual in the linearized thermoelasticity. The arguments of this section can be adapted without difficulties to the linear elasticity by virtue of the Korn inequality whenever we assume taht the elasticity tensor defines a positive functions on the strains. 


\section{Exponential decay of solutions}

The aim of this section is to prove that the solutions of the problem determined by (2.1), (2.2), (2.6) and (2.7), when the supply terms vanish, decay in an exponential way. To be precise we are going to prove that there exist two positive constants $M$ and $\omega$ independent of the initial data such that

$$
E(t) \leq M E(0) \exp (-\omega t)
$$

To show this result we need to consider a new function

$$
G(t)=\frac{\tau_{1}}{2} \int_{B} C_{i j k l} u_{i, j} u_{k, l} d v
$$

We note that

$$
\dot{G}(t)=-\int_{B} \rho \ddot{u}_{i} u_{i} d v+\int_{B} \gamma_{i j}\left(\theta+\tau_{1} \dot{\theta}\right) u_{i, j} d v-\int_{B} C_{i j k l} u_{i, j} u_{k, l} d v
$$

After the use of the arithmetic-geometric mean inequality and the Poincaré inequality we see that there exists a positive constant $M_{1}$ such that

$$
\dot{G}(t) \leq M_{1} \int_{B}\left(\rho \ddot{u}_{i} \ddot{u}_{i}+\theta^{2}+|\dot{\theta}|^{2}\right) d v-\frac{1}{2} \int_{B} C_{i j k l} u_{i, j} u_{k, l} d v .
$$

We shall denote

$$
E_{\epsilon}(t)=E(t)+\epsilon G(t)
$$

It is clear that whenever $\epsilon$ is positive, the following inequalities

$$
\alpha_{1} E_{\epsilon}(t) \leq E(t) \leq \alpha_{2} E_{\epsilon}(t),
$$

hold, where $\alpha_{1}, \alpha_{2}$ are two calculable positive constants. Thus, the inequalities (4.6) allow us to say that whenever $\epsilon$ is positive, the functions $E(t)$ and $E_{\epsilon}(t)$ define equivalent measures.

In view of the equality (3.6) and the estimate (4.4) we see

$$
\begin{gathered}
\dot{E}_{\epsilon}(t) \leq-\int_{B}\left(\left(\tau_{0}-\epsilon M_{1}\right) \rho \ddot{u}_{i} \ddot{u}_{i}+\left(\tau_{1}-\tau_{0}\right) C_{i j k l} \dot{u}_{i, j} \dot{u}_{k, l}\right. \\
\left.+\left(\left(\tau_{1}-\tau_{0}\right) \rho c_{E}-\epsilon M_{1}\right)|\dot{\theta}|^{2}+k_{i j} \theta_{, i} \theta_{, j}-\epsilon M_{1} \theta^{2}+\frac{1}{2} C_{i j k l} u_{i, j} u_{k, l}\right) d v .
\end{gathered}
$$

From last estimate and after the use of the Poincaré inequality we may select $\epsilon>0$ small enough to conclude the existence of a positive constant $M_{2}$ such that

$$
\dot{E}_{\epsilon}(t) \leq-M_{2} E(t)
$$

whenever $\epsilon$ is small enough, but positive. Inequality (4.8) combined with the first estimate of (4.6) imply that

$$
\dot{E}_{\epsilon}(t) \leq-M_{2} \alpha_{1} E_{\epsilon}(t)
$$

This inequality implies that

$$
\dot{E}_{\epsilon}(t) \leq E_{\epsilon}(0) \exp \left(-M_{2} \alpha_{1} t\right)
$$

This bound combined with (4.6) imply the estimate (4.1). Therefore, we have proved: 
Theorem 4.1. Let $\left(u_{i}, \theta\right)$ be a solution of the initial-boundary-value problem determined by the system (2.1), (2.2), the initial condition (2.6) and the boundary conditions (2.7) with null supply terms $\left(b_{i}=r=0\right)$. Then, the solutions satisfy the estimate (4.1) where the function $E(t)$ is defined at (3.5).

Again, the analysis of this section can be adapted directly to the linear thermoelasticity.

\section{Spatial Behavior}

In this section we obtain a Phragmén-Lindelöf alternative for the solutions of the homogeneous version of the system of equations $(2.1),(2.2)$. To do that we are going to change the domain where the problem is proposed. In this section $B$ will be a semiinfinite cylinder $B=[0, \infty) \times D$, where $D$ is a two dimensional bounded domain smooth enough to apply the divergence theorem. We also consider appropriate boundary and initial conditions. We assume that null initial conditions

$$
u_{i}(\mathbf{x}, 0)=\dot{u}_{i}(\mathbf{x}, 0)=\theta(\mathbf{x}, 0)=\dot{\theta}(\mathbf{x}, 0)=0, \mathbf{x} \in B .
$$

We also suppose that

$$
u_{i}(\mathbf{x}, t)=\theta(\mathbf{x}, t)=0, \mathbf{x} \in[0, \infty) \times \partial D, t>0
$$

and

$$
u_{i}\left(0, x_{2}, x_{3}, t\right)=f_{i}\left(x_{2}, x_{3}, t\right) \quad \theta(\mathbf{x}, t)=g\left(x_{2}, x_{3}, t\right), \mathbf{x} \in\{0\} \times D, \quad t>0 .
$$

In this case the analysis starts by considering the function

$$
H_{\omega}(z, t)=-\int_{0}^{t} \int_{D(z)} \exp (-2 \omega t) \Phi d a d s
$$

where $D(z)=\left\{\mathbf{x} \in B, x_{1}=z\right\}$,

$$
\Phi=\left(C_{i 1 k l}\left(u_{k, l}+\tau_{1} \dot{u}_{k, l}\right)-\gamma_{i 1}\left(\theta+\tau_{1} \dot{\theta}\right)\right)\left(\dot{u}_{i}+\tau_{0} \ddot{u}_{i}\right)+k_{i 1} \theta_{, i}\left(\theta+\tau_{1} \dot{\theta}\right) .
$$

An use of the divergence theorem with the initial and boundary conditions shows that

$$
H_{\omega}(z+h, t)-H_{\omega}(z, t)=\frac{\exp (-2 \omega t)}{2} \int_{B(z+h, z)} \Upsilon_{1} d v+\int_{0}^{t} \int_{B(z+h, z)} \exp (-2 \omega s) \Upsilon_{2} d v d s
$$

where $B(z+h, z)=\left\{\mathbf{x} \in B, z<x_{1}<z+h\right\}$, and

$$
\begin{gathered}
\Upsilon_{1}=\rho \dot{u}_{i} \dot{u}_{i}+C_{i j k l} u_{i, j} u_{k, l}+\tau_{0} \tau_{1} C_{i j k l} \dot{u}_{i, j} \dot{u}_{k, l}+\rho c_{E} \theta^{2}+\tau_{0} \tau_{1} \rho c_{E}|\dot{\theta}|^{2} \\
+\tau_{1} k_{i j} \theta_{, i} \theta_{, j}+2 \tau_{0} C_{i j k l} \dot{u}_{i, j} u_{k, l}+2 \rho c_{E} \tau_{0} \theta \dot{\theta}
\end{gathered}
$$

and

$$
\begin{aligned}
\Upsilon_{2}=\omega & \Upsilon_{1}+\tau_{0} \rho \ddot{u}_{i} \ddot{u}_{i}+\left(\tau_{1}-\tau_{0}\right) C_{i j k l} \dot{u}_{i, j} \dot{u}_{k, l} \\
& +\left(\tau_{1}-\tau_{0}\right) \rho c_{E}|\dot{\theta}|^{2}+k_{i j} \theta_{, i} \theta_{, j} .
\end{aligned}
$$


In particular, when

$$
\lim _{z \rightarrow \infty} H_{\omega}(z, t)=0
$$

we see that

$$
-H_{\omega}(z, t)=\frac{\exp (-2 \omega t)}{2} \int_{B(\infty, z)} \Upsilon_{1} d v+\int_{0}^{t} \int_{B(\infty, z)} \exp (-2 \omega s) \Upsilon_{2} d v d s .
$$

From (5.6), it follows that

$$
\frac{\partial H_{\omega}}{\partial z}=\frac{\exp (-2 \omega t)}{2} \int_{D(z)} \Upsilon_{1} d v+\int_{0}^{t} \int_{D(z)} \exp (-2 \omega s) \Upsilon_{2} d v d s
$$

Our next step consists to evaluate the absolute value of the function $H_{\omega}$ in terms of its spatial derivative.

Because of the use of the arithmetic geometric mean inequality we see that there exists a positive constant $K_{\omega}$ such that ${ }^{3}$

$$
|\Phi| \leq K_{\omega} \Upsilon_{2}
$$

Therefore we obtain that

$$
\left|H_{\omega}\right| \leq K_{\omega} \frac{\partial H_{\omega}}{\partial z}
$$

This inequality is classical in the studies on the spatial stability and yields a PhragménLindelöf alternative (see [4]). More precisely, if there exists $z_{0} \geq 0$ such that $H_{\omega}\left(z_{0}, t\right)>$ 0 , then the solution satisfies the estimate

$$
H_{\omega}(z, t) \geq H_{\omega}\left(z_{0}, t\right) \exp \left(K_{\omega}^{-1}\left(z-z_{0}\right)\right), \quad z \geq z_{0} .
$$

This estimate gives information in terms of the measure defined in the cylinder. Indeed, it follows that

$$
\frac{\exp (-2 \omega t)}{2} \int_{B(z+h, z)} \Upsilon_{1} d v+\int_{0}^{t} \int_{B(z+h, z)} \exp (-2 \omega s) \Upsilon_{2} d v d s
$$

tends to infinity exponentially fast when $h$ is increasing. On the contrary, when $H_{\omega}(z, t) \leq 0$, for every $z \geq 0$, it follows that the solution decays and we can obtain an estimate of the form

$$
-H_{\omega}(z, t) \leq-H_{\omega}(0, t) \exp \left(-K_{\omega}^{-1} z\right), \quad z \geq 0 .
$$

This inequality implies that $H_{\omega}(z, t)$ tends to zero as $z$ goes to infinity. Furthermore in view of this estimate, it is clear that

$$
E_{\omega}(z, t) \leq E_{\omega}(0, t) \exp \left(-K_{\omega}^{-1} z\right), \quad z \geq 0
$$

where

$$
E_{\omega}(z, t)=\frac{\exp (-2 \omega t)}{2} \int_{B(\infty, z)} \Upsilon_{1} d v+\int_{0}^{t} \int_{B(\infty, z)} \exp (-2 \omega s) \Upsilon_{2} d v d s, \quad z \geq 0
$$

Finally we can state:

\footnotetext{
${ }^{3}$ It is worth noting that the best value for the $K_{\omega}$ involves the study of a very cumbersome system of nonlinear equations.
} 
Theorem 5.1. Let $\left(u_{i}, \theta\right)$ be a solution of the initial-boundary-value problem determined by the system (2.1), (2.2), the initial condition (5.1) and the boundary conditions (5.2), (5.3) with null supply terms $\left(b_{i}=r=0\right)$. Then, either this solution satisfies the growth estimate (5.14) or it satisfies the decay estimate

$$
E_{\omega}^{*}(z, t) \leq E_{\omega}(0, t) \exp \left(2 \omega t-K_{\omega}^{-1} z\right), \quad z \geq 0 .
$$

where

$$
E_{\omega}^{*}(z, t)=\frac{1}{2} \int_{B(\infty, z)} \Upsilon_{1} d v+\int_{0}^{t} \int_{B(\infty, z)} \Upsilon_{2} d v d s, z \geq 0 .
$$

This kind of behaviour is typical in several thermoelastical problems [13].

\section{Acknowledgments}

The investigation reported in this paper is supported by the project project "Análisis Matemático de Problemas de la Termomecánica" (MTM2016-74934-P)(AEI/FEDER, UE) of the Spanish Ministry of Economy and Competitiveness. The author thanks to the anonymous referee his useful suggestions concerning this submission.

\section{Compliance with ethical standards. Conflict of interest}

The author declares that he has no conflict of interest.

\section{References}

[1] F. Bofill, R. Quintanilla "Some qualitative results for the theory of thermomicrostretch elastic solids" Int. Jour. Eng. Sci. 33, (1995) 2115-2125

[2] C. Cattaneo "Sulla conduzioini del calore" Atti Sem. mat. Fis. Univ. Modena, 3 (1948), 83-101.

[3] S. Chirita "Saint-Venant's principle in linear elasticity" Jour. Thermal Stresses, 18 (1995), 485-496.

[4] J. N. Flavin, R. J. Knops, L. E. Payne, "Decay estimates for the constrained elastic cylinder of variable cross-section" Quart. Appl. Math. 47 (1989), 325-350.

[5] A. E. Green, K. Lindsay "Thermoelasticity" J. Elast.2 (1972) , 1-7.

[6] A. E. Green P. M. Naghdi "On undamped heat waves in elastic solids" J. Thermal Stresses 15 (1992) 252-264

[7] A. E. Green P. M. Naghdi "Thermoelasticity without energy dissipation" J. Elast. 31 (1993) 189-208.

[8] C.O. Horgan, R. Quintanilla "Spatial behaviour of solutions of the dual-phase-lag heat equation" Mathematical Methods Applied Sciences, 28, (2005) 43-57

[9] D. Iesan, R. Quintanilla "On a theory of thermoelasticity with microtemperatures" J. Thermal Stresses 23, (2000) 199-216

[10] Y. Jun Yu, Zhang-Na Xue and Xiao-Gen Tian "A modified Green-Lindsay thermoelastidcity with strain rate to eliminate discontinuity." Meccanica 53 (2018) 2543-2554. 
[11] M.C. Leseduarte, A. Magaña, R. Quintanilla "On the time decay of solutions in porous-thermo-elasticity of type II" Discrete and Continuous Dynamical Systems, Series B, 13, (2010) 375-391

[12] M.C. Leseduarte, R. Quintanilla "On the spatial behavior in type III thermoelastodynamics" Jour. Appl. Math. Physics (ZAMP) 65, (2014), 165-177

[13] M.C. Leseduarte, R. Quintanilla "Spatial behavior in high order partial differential equations" Mathematical Methods Applied Sciences, 41, (2018) 2480-2493

[14] H. Lord, Y. Shulman "A generalized dynamic theory of thermoelasticity" J. Mech. Phys. solids 15 (1967), 299-309.

[15] C. B. Navarro, R. Quintanilla "On existence and uniquenes in incremental thermoelasticity" Jour. Appl. Math. Physics (ZAMP) 35, (1984), 206-215

[16] R. Quintanilla "End effects in thermoelasticity" Mathematical Methods in Applied Sciences, 24,(2001) 93-102

[17] R. Quintanilla, R. Racke "Stability in thermoelasticity of type III" Discrete and Continuous Dynamical Systems B, 3, (2003) 383-400.

[18] R. Quintanilla, B. Straughan "Growth and uniqueness in thermoelasticity" Proceedings of the Royal Society of London, Series A, 456, (2000) 1419-143

[19] A. J. C. B. Saint-Venant "Mémoire sur la torsion des prismes" Mémoires présentés pour divers Savants a Ácadémie des Sciences de l'Institut Impérial de France, 14 (1853), 233-560

[20] A. J. C. B. Saint-Venant "Mémoire sur la flexion des prismes" J. Math. Pures Appl 1 (Ser. 2) (1856) 89-189. 